\title{
Author Correction: Low frequency transcranial electrical stimulation does not entrain sleep rhythms measured by human intracranial recordings
}

\author{
Belen Lafon (10) ', Simon Henin (10) 2,3, Yu Huang (10) ', Daniel Friedman (10) 2,3, Lucia Melloni (1) 2,3,4, \\ Thomas Thesen (10) ${ }^{3,5}$, Werner Doyle 2,6 , György Buzsáki (i] ${ }^{3,7}$, Orrin Devinsky (i) ${ }^{2,3}$, Lucas C. Parra (i) ${ }^{1}$ \& \\ Anli Liu (1) 2,3
}

Correction to: Nature Communications https://doi.org/10.1038/s41467-017-01045-x, published online 31 October 2017

It has come to our attention that we did not specify whether the stimulation magnitudes we report in this Article are peak amplitudes or peak-to-peak. All references to intensity given in $\mathrm{mA}$ in the manuscript refer to peak-to-peak amplitudes, except in Fig. 2, where the model is calibrated to $1 \mathrm{~mA}$ peak amplitude, as stated. In the original version of the paper we incorrectly calibrated the computational models to $1 \mathrm{~mA}$ peak-to-peak, rather than $1 \mathrm{~mA}$ peak amplitude. This means that we divided by a value twice as large as we should have. The correct estimated fields are therefore twice as large as shown in the original Fig. 2 and Supplementary Fig. 11. The corrected figures are now properly calibrated to $1 \mathrm{~mA}$ peak amplitude. Furthermore, the sentence in the first paragraph of the Results section 'Intensity ranged from 0.5 to $2.5 \mathrm{~mA}$ (current density $0.125-0.625 \mathrm{~mA} \mathrm{~mA} / \mathrm{cm}^{2}$ ), which is stronger than in previous reports', should have read 'Intensity ranged from 0.5 to $2.5 \mathrm{~mA}$ peak to peak (peak current density $0.0625-0.3125 \mathrm{~mA} / \mathrm{cm}^{2}$ ), which is stronger than in previous reports.' These errors do not affect any of the Article's conclusions. Correct versions of Fig. 2 and Supplementary Fig. 11 are presented below as Figs. 1, 2.

\footnotetext{
${ }^{1}$ Department of Biomedical Engineering, City College of New York, 160 Convent Ave, New York, NY 10031, USA. ${ }^{2}$ New York University Comprehensive Epilepsy Center, 223 East 34th Street, New York, NY 10016, USA. ${ }^{3}$ Department of Neurology, New York University School of Medicine, 240 East 38 th St, 20th Floor, New York, NY 10016, USA. ${ }^{4}$ Department of Neuroscience, Max Planck Institute for Empirical Aesthetics, Gruneburgweg 14, 60322 Frankfurt am Main, Germany. ${ }^{5}$ Department of Physiology and Neuroscience, St. George's University, St. George's, Grenada. ${ }^{6}$ Department of Neurosurgery NYU School of Medicine, 530 1st Avenue, Suite 7W, New York, NY 10016, USA. ${ }^{7}$ New York University Neuroscience Institute, 450 East 29th St, New York, NY 10016, USA. These authors contributed equally: Belen Lafon, Simon Henin. These authors jointly supervised this work: Lucas C. Parra, Anli Liu. The original article can be found online at https://doi.org/10.1038/s41467-017-01045-x.Correspondence and requests for materials should be addressed to A.L. (email: anli.liu@nyumc.org)
} 
a

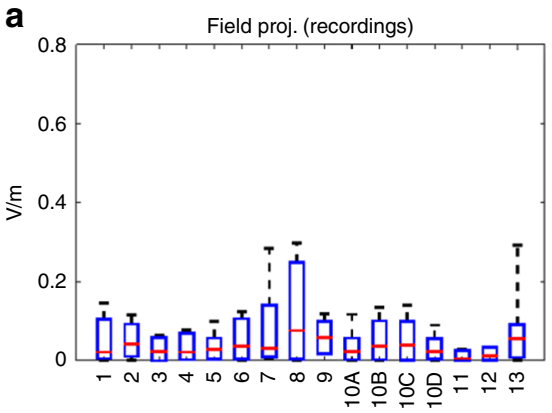

Subject b

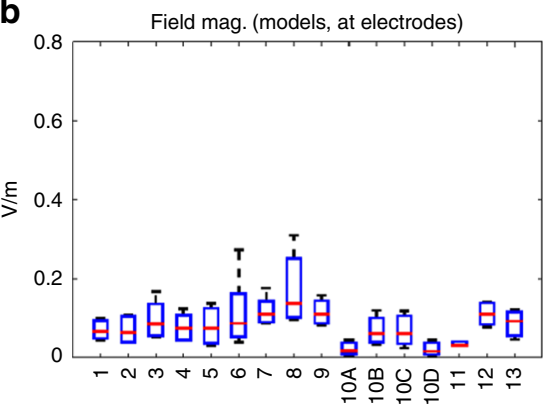

Subject
C

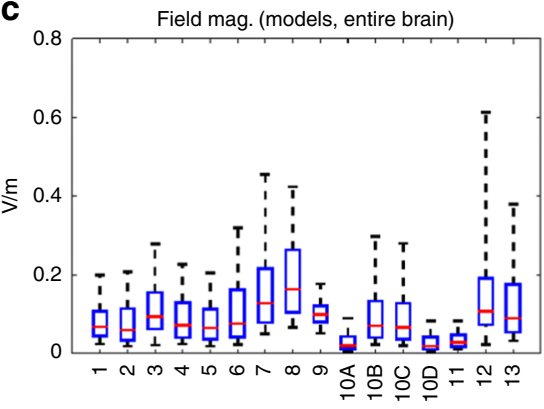

Subject

Fig. 1
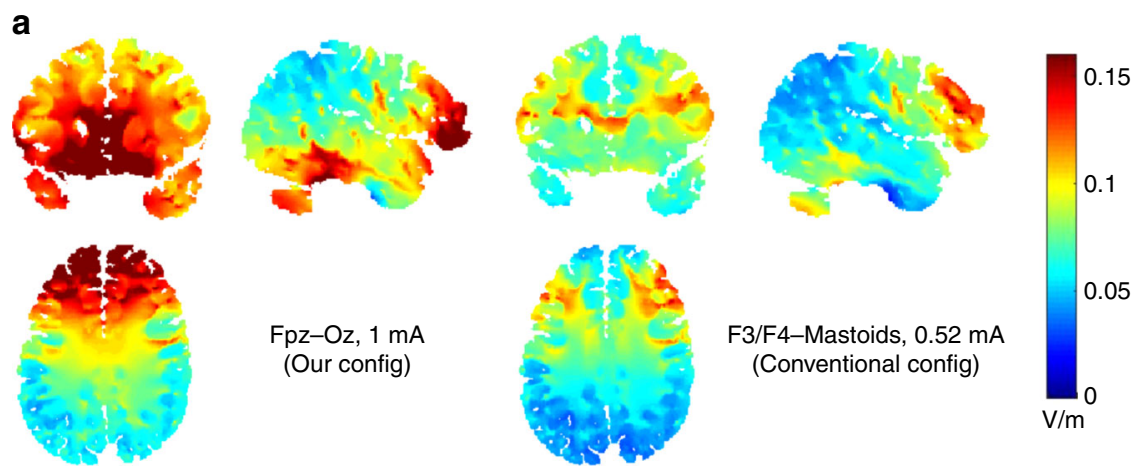

b

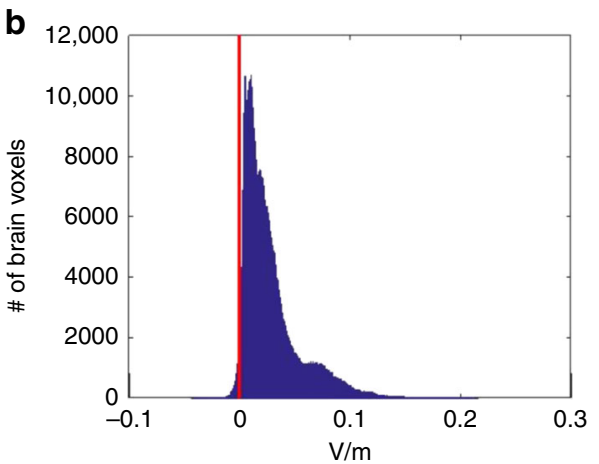

Fig. 2

Published online: 28 February 2018

(c) Open Access This article is licensed under a Creative Commons Attribution 4.0 International License, which permits use, sharing, adaptation, distribution and reproduction in any medium or format, as long as you give appropriate credit to the original author(s) and the source, provide a link to the Creative Commons license, and indicate if changes were made. The images or other third party material in this article are included in the article's Creative Commons license, unless indicated otherwise in a credit line to the material. If material is not included in the article's Creative Commons license and your intended use is not permitted by statutory regulation or exceeds the permitted use, you will need to obtain permission directly from the copyright holder. To view a copy of this license, visit http://creativecommons.org/licenses/by/4.0/.

(C) The Author(s) 2018 